Mario H. Ramírez Díaz ${ }^{1}$, Miguel Olvera Aldana ${ }^{2}$

${ }^{1}$ Centro de Investigación en Ciencia Aplicada y Tecnología Avanzada, Instituto Politécnico Nacional, Av. Legaria \# 694, Col. Irrigación Del. Miguel Hidalgo, CP 11500, México D. F.

${ }^{2}$ Escuela Superior de Cómputo, Instituto Politécnico Nacional, Av. Juan de Dios Batiz s/n esq. Miguel Othón de Mendizábal. Col. Linda vista. CP 07738, México DF.

E-mail: mramirezd@ipn.mx

PACS: $01.30 . \mathrm{Vv}$

\section{Infrared Thermal Imaging Fundamentals, Research and Applications}

\author{
Vollmer, M. \& Möllman, K.-P.593 pp., edited by \\ WYLEY-VCH, Germany, 2010. ISBN: 978-3- \\ 527-40717-0.
}

In the first part the book made a historical reference about the thermal images evolution and his forward into the science until current days.

The book structure gives to the reader three options:

- The first part is dedicated to understanding of some phenomena and their applications in different fields, both quantitative and qualitative way.

- The second part is directed to teachers and students in college level in physics area; because this technique is a good tool to visualization several physic phenomena.

- Finally shows some lines of research that uses IR images.

The book contains ten chapters and can be read in separate. The authors organize the material as:

Chapter's 1-3. Was provides information about physics principles in IR images and their historical context.

Chapter 4. Was provides basics aspects about heat transference, like radiation, conduction and convection and their applications.

Chapter's 5-9. Each chapter studies different applications of IR images, since Teach until industrial applications.

Chapter 10. The authors made some thoughts about topics to research as like medicine or sports.

In the chapter one is detailed the preliminary concepts of optics, beginning geometrical optics, Law of Snell and Fresnell equations until concepts of radiation, all with a mathematical develop in an understanding level accessible.

In particular, the authors made an analysis of Blackbody radiation and form that was studied it by Plack and his famous equation, furthermore the comparative with Law of Wien and Law of Stephan-Bolztmann; this comparative serves to have an accurate idea how calibrate IR cameras. On the other hand, shows a practical example about how adjust distance to a better use the camera. Also discusses the factors that influence the images like temperature changes as well as materials used.
In chapter two detail the characteristics of IR camera components, doing emphasis in detector, doing an important studio of the physic properties measurable. Properties of thermic detectors and photonic detectors were studied among others.

Furthermore include a complete studio obtaining and processing of IR images, as the interpretation and adjust of the results.

In the chapter three deepens on advanced methods in IR images. Was talk about different kind of filters and their influence on object signals. Shows basic aspects of spectrometers and exemplifies a hyperspectral imaging instrument. Furthermore was detailed the IR images process, from basic methods until advanced methods of images processing. The chapter four speak about basic principles of heat transfer like conduction, convection and radiation. In this chapter discussed the relevance of measured surface temperatures of objects like buildings, electrical components under load, animal or humans due to their emitted IR radiation using IR cameras. Especially interesting is the section 4.3, show selected examples for heat transfer problems. The Table 4.3 shows parameters/conditions referring to standard situations in thermography like a introduction to standard situations witch resemble typical measurement situations including limiting cases. The selected examples in detailed are: Conduction within Solids: The Biot Number, Steady-State Heat Transfer through One-Dimensional Walls and UValue, Heat Transfer through Windows, Steady-State Heat Transfer in Two and Three Dimensional Problems: Thermal Bridges, Dew Points Temperatures, Transient Effects: Heating and Cooling of Objects, Heat Capacity and Thermal Diffusivity, Short Survey of Quantitative Treatments of Time-Dependent Problems, Typical Time Constants for Transient Thermal Phenomena, Cooling Cube Experiment, Theoretical Modeling of Cooling of Solid Cubes, Time Constant for Different Objects, Some Thoughts on Validity of Newton's Law, Theoretical Cooling Curves, Relative Contributions of Radiation and Convection, and end the chapter with Some Experiments: Heating and Cooling Light Bulbs.

At long of chapter the authors develop theoretical concepts clearly through equations and respective graphics. The illustrations has an excellent quality and 
Mario H. Ramírez Díaz, Miguel Olvera Aldana

allowed see the phenomena exampled. Especially important to physics education result the Thoughts on the validity of Newton's Law, doing an accurate description of cooling process introducing the radiation contribution in its original nonlinear form.

Chapter five is vital to teaching because talking about Basic Applications for Teaching: Direct Visualization of Physics Phenomena. The importance of this chapter is notorious if remember that infrared thermal imaging allows quantitative and qualitative imaging of multitude of phenomena and processes in physics. The chapter begins with a review of transformation of mechanical energy into heat, taking on count that a very important field for IR imaging in physics education concerns the visualization of mechanical phenomena involving friction. It shows images of low and high resolution cameras of the braking of motorbike with blocked tires to show that the sliding friction cause temperature rises during braking for example.

This chapter uses illustrative examples like Tennis or the human balance for inelastic collisions and shows the temperature rise. The figures in the chapter illustrate clear thermal physics phenomena like energy dissipation, homogeneous heating and thermal conductivities, this figures shows IR images very useful in the teaching of heat interchange. Other examples taken of the ordinary life are heating of cheese cubes and cooling of bottles to shows heating and cooling of several objects. A curious detail in the figures is the fact that authors use them own image to shows some phenomena like IR images of people, transmission of window glass or diffuse reflection.

In the chapters 6 and 7 are studied several applications of IR images some of them are: Building and Infrastructure (all chapter six is dedicated to this topic), Industrial Application (Especially detection of gases). Both applications are very useful for engineering and architecture students, again the IR images and its use are developed on a very didactic way.

The chapter eight deals about microsystems engineering. Obviously thermography play an important role for thermal characterization of different microsystems like microfluidic systems or systems wich utilize electric to thermal energy conversion during operation.

This chapter have an especially interest because the thermography of microsystems poses a number of problems which are not usually encountered when studying macroscopic objects. The chapter provides a set of applications, since microreactors and microsensors until cryogenic actuators, all of the illustrative uses of IR images presents in a very didactic form to engineering or applied physics students.

Finally the chapter ten gives selected applications of IR images in several fields, all of them of especially interest to physics and engineering students like sports, arts or musical instruments. 